\title{
Editorial. Nationalismes et construction européenne
}

Daniel Hermant

\section{(2) OpenEdition \\ 1 Journals}

Édition électronique

URL : http://journals.openedition.org/conflits/658

DOI : $10.4000 /$ conflits.658

ISSN : $1777-5345$

Éditeur :

CCLS - Centre d'études sur les conflits lilberté et sécurité, L'Harmattan

Édition imprimée

Date de publication : 22 octobre 1992

ISSN : 1157-996X

Référence électronique

Daniel Hermant, « Editorial. Nationalismes et construction européenne », Cultures \& Conflits [En ligne], 07 | automne 1992, mis en ligne le 07 janvier 2003, consulté le 30 mars 2021. URL : http://

journals.openedition.org/conflits/658 ; DOI : https://doi.org/10.4000/conflits.658

Ce document a été généré automatiquement le 30 mars 2021.

Creative Commons License 


\title{
Editorial. Nationalismes et construction européenne
}

\author{
Daniel Hermant
}

1 L'Europe n'est-elle pas en train de faire le lit des contestations nationales ou micronationales, d'ouvrir la boîte de Pandore des nationalismes? Cette affirmation peut paraître paradoxale, surtout au moment où face à la désintégration de l'Europe communiste il semble raisonnable de se féliciter des succès de l'intégration européenne. Paix à l'Ouest, guerre à l'Est donc, mais derrière cette rhétorique un peu trop affirmée ne faut-il pas lire une secrète inquiétude, comme si entre ces deux évolutions, existaient des passerelles voire une logique commune? Que nous montre l'Est : un échec économique spectaculaire, doublé d'un changement brutal de système politique qui entraîne d'abord la décomposition du bloc impérial soviétique ; puis dans une deuxième étape provoque l'éclatement des États successeurs ravalés au rang de micro-empires! Dans cette réduction progressive des échelles, dans ce jeu sans cesse renouvelé des logiques identitaires dont on se demande l'issue, ne peut-on trouver un principe général qui pourrait à l'occasion fonctionner à l'Ouest ${ }^{1}$ ? Ce problème, qui est au coeur du présent numéro de Cultures et Conflits, peut se formuler de la manière suivante : coincé par la construction européenne entre un niveau supranational qui lui ôte une à une ses compétences et un niveau régional qui réclame une autonomie sans cesse plus grande, l'État-nation - ossature de notre société politique - est à la fois délégitimé et contesté, en tout état de cause fragilisé2.

2 A partir de l'examen des positions et de l'action d'acteurs politiques quelquefois marginaux mais placés au c ?ur de la contestation identitaire : partis d'extrême droite, Ligues Lombardes, mouvements régionalistes, organisations clandestines à vocation indépendantiste, les contributions que nous réunissons cherchent à tester la réalité de ces craintes, à voir si ce mécanisme de corrosion interne correspond à un simple effet de raisonnement ou au contraire s'il est déjà à l'oeuvre dans la Communauté européenne ${ }^{3}$.

Choix européens 
3 La position à l'égard de l'Europe des partis ou des organisations que nous venons d'énumérer peut servir de point de départ à notre enquête, bien que la construction européenne ne soit entrée dans le champ de leurs préoccupations qu'assez tardivement ${ }^{4}$. Nous distinguerons deux groupes : le premier est anti-européen, il se recrute dans les partis d'extrême droite, dans les organisations clandestines ou les mouvements politiques qui les soutiennent. Le Front national dénonce la "dérive vers une entité supranationale qui passe par l'éclatement de la nation" et réclame dans la foulée "un Empire européen des patries fédérées et non homogènes" 5 . J. M. Le Pen stigmatise les "Euristocrates" aux ordres de "l'oligarchie internationale cosmopolite"6 et va jusqu'à imaginer qu'il y a complot contre la nation et volonté de dissoudre l'identité nationale. On trouve une attitude aussi tranchée sur des arguments différents dans les mouvements clandestins et plus largement dans la mouvance nationaliste. Le FLNCcanal historique parle, à propos de Maastricht, d'une "union d'États-bunker qui continuent à nier des nationalités et des peuples qui constituent son espace"7, le Sinn Fein de "super-État centralisé où la politique sera décidée par une bureaucratie non élue"s. Sur le bord opposé, les partis unionistes protestants disent : "non à Maastricht, non à Delors".

Un deuxième groupe voit au contraire dans l'Europe une dimension indispensable à toute solution des problèmes basque, corse, irlandais ou italien. L'Irlande représente sans doute le cas emblématique de cette vision plus raffinée des problèmes de régionalisme. L'idée de la dimension incontournable de l'internationalisation du débat y chemine, nous dit A. Guelke, depuis plus de 40 ans. Cette conviction s'est imposée chez presque tous les partenaires : on la trouve au Nord comme au Sud, chez les États comme chez les partis, chez les catholiques comme chez les protestants. On pourrait retrouver des positions similaires en Espagne, avec la position du PNV très ouverte sur l'Europe $^{10}$, en Corse où le MPA a également dit oui à l'Europe, ou en Italie avec la position ultra-européenne de la Ligue lombarde.

5 Pourtant cette belle simplicité se brouille dès que se précise l'observation, car l'attitude à l'égard de l'Europe est un sujet de division à l'intérieur de la mouvance nationaliste. A. Guelke et E. Picard soulignent avec force que le choix européen ne recouvre pas la division partisane, aux deux extrémités du spectre politique irlandais, unionistes protestants et nationalistes catholiques sont contre l'Europe et inversement, au centre, des liens sont créés entre unionistes libéraux de l'Alliance Party et nationalistes constitutionnels du SDLP favorable à l'Europe ${ }^{11}$. En Corse, le mouvement nationaliste se divise sur Maastricht : le MPA vote pour, l'ANC vote contre, les autres tendances avec des nuances - l'UPC, ACN, et I Verdi corsi - prônent l'abstention. Au Pays basque, le nationalisme têtu d'ETA et d'HB récuse l'Europe alors que le PNV en est un chaud partisan, puisque sa branche basco-française déclare "voter oui à Maastricht est un impératif". Le reste des parfis basques en France est divisé, Eusko Alkartasuna et Euskal Batasuna votant également oui, alors que le mouvement nationaliste EMA et PaxtaOldartzen sont contre. Quand au Front national ou aux Republikaner allemands, ils sont peu cohérents, ou plutôt opportunistes dans leurs prises de position à l'égard de l'Europe. Le Front national, par exemple, change complètement d'attitude entre 1984, où il était en faveur d'une Europe puissante et 1989, où la relance communautaire lui paraît menacer l'identité nationale. En 1983, le programme des Republikaner dénonçait les "égoïsmes nationaux" et était plutôt favorable à l'Europe. A partir de 1987, ils voient avant tout dans l'Europe un obstacle à la réunification qui est leur priorité, mais une 
fois celle-ci réalisée, ils maintiennent cependant leur opposition à l'Europe présentée comme la source de tous les maux.

6 Sur un autre plan, les logiques autocentrées que développent ces acteurs politiques visent au développement d'entités closes sur elles-mêmes et ne peuvent qu'entraîner des conflits opposant les partis "identitaires nationaux" entre eux ou les partis "identitaires nationaux" et "identitaires régionaux". Pour le Front national, la France doit être "le moteur de l'Europe", alors que pour les Republikaner ce rôle revient à l'Allemagne $^{12}$. A cette concurrence pour l'hégémonie, s'ajoutent les positions divergentes sur le régionalisme. A. d'Appollonia rappelle la stratégie en zigzag du Front national au sein du groupe des droites européennes au Parlement de Strasbourg où cette formation soutient le Vlaams block flamand et le groupe wallon Agir, tous les deux séparatistes, alors qu'elle est en désaccord avec le Front national belge qui pourtant défend l'unité de la Belgique $!^{13}$ En Italie, le populisme national du MSI se heurte au populisme régionaliste des Ligues.

7 Ces positionnements contradictoires et assez opportunistes s'expliquent par la variété des acteurs convoqués - la distance est grande entre une organisation clandestine et un parti politique à vocation nationale - et surtout par la dimension à leurs yeux, secondaire, de la question européenne. Aussi n'est-il pas surprenant que nous n'obtenions qu'une constellation d'opinions dessinées en creux. Pour faire système, ces réponses doivent être resituées par rapport à l'idéologie et à l'activité principale de ces acteurs, donc par rapport à ce qui les structure réellement.

Affrontements binaires

8 L'attitude des mouvements de libération nationale et des organisations clandestines opposés à l'Europe dessine une première configuration générale d'acteurs dont l'unité réside dans une logique d'affrontement dont le terme est la création de nouveaux États. La stratégie d'indépendance développée par ces groupes s'appuie sur la présence d'un adversaire bien identifié (la France ou l'Espagne par exemple), fait une place importante à l'action directe et à la violence, mais ignore l'Europe. Ces caractéristiques expliquent que le débat sur la place prééminente de la lutte armée dans le processus d'indépendance soit toujours central dans ces mouvements ${ }^{14}$ D'ailleurs, pour analyser le répertoire d'action d'organisations clandestines comme l'ETA, l'IRA, le FLNC, l'observateur doit décrypter la batterie de critères stratégico-politiques (clandestinité, dialectique légal/illégal, logique provocation/répression) avec laquelle ces acteurs replacent leur action dans le cadre dynamique d'un affrontement et rendent compte des logiques de mobilisation populaire sur lesquelles ils appuient leur légitimité. Géographiquement, les effets de la violence sont circonscrits : le national est atteint par l'intermédiaire du local, et l'international trop lointain est ignoré. La violence interne est rarement délocalisée de la région vers l'Europe ${ }^{15}$. Celle-ci donc est absente ou n'apparaît qu'épisodiquement comme une ombre ou un moyen. La réorientation des attentats des FLNC sur les thèmes de la lutte contre la spéculation immobilière ou la Mafia n'est pas une réponse à l'impact d'une Europe anarchique, ultra-libérale permettant l'entrée sans contrôle des capitaux et des hommes, mais une nouvelle justification idéologique de la violence.

9 Le discours qui structure ces actes est très militant et orienté essentiellement contre l'État-nation. Sur le plan économique, les acteurs présentent les régions comme l'envers et les concurrents des États nationaux, leur modèle obstacle. Une vive rhétorique dénonce la politique du centre par rapport à la périphérie et propose 
comme solution l'instauration d'un système socialiste. Le "secondu quaternu" de $\mathrm{l}^{\prime} \mathrm{aCN}^{16}$ ou le document d'orientation d'Herri Batasuna ${ }^{17}$ contiennent tous deux des développements sur le socialisme qu'il est nécessaire d'instaurer, choix qui est également celui du Sinn Fein. Cette rhétorique s'enracine dans le discours de décolonisation qui dominait au moment où ces mouvements se sont constitués et en réadapte à peine les principales articulations par une formulation en termes de différentiel de prospérité, de chances de croissance qui voile mal l'archaïsme du discours. Dans ce schéma, l'Europe n'est qu'un élément secondaire. Pourtant au Pays basque, en Corse ou en Irlande du Nord, l'entrée dans la Communauté est crainte car l'Europe est perçue comme un élément amplifiant les effets négatifs de la politique des États : l'Europe des marchands renforcerait les inégalités, marginaliserait encore plus l'Irlande du Nord, favoriserait le démantèlement de la sidérurgie basque et assignerait à la Corse "la mission de centre de repos et de loisirs pour les privilégiés de la fortune" ${ }^{18}$. Il est intéressant d'opposer à ce discours celui très pro-européen des Ligues Lombardes qui inverse la critique, dénonçant l'archaïsme de l'État central italien générateur pour les régions riches de l'Italie du Nord, de contraintes économiques très lourdes.

10 Sur le plan culturel, point fort de toutes les démarches identitaires, l'objectif est de rétablir la culture nationale dans toute sa pureté. Cette attitude est commune aux partis d'extrême droite et aux organisations clandestines, et se retrouve également, malgré quelques précautions de langage, au sein des Ligues Lombardes ${ }^{19}$. Pour les partis d'extrême droite, elle prend plutôt l'allure d'une dénonciation du laxisme des décideurs face à l'invasion étrangère, pour les organisations clandestines elle s'appuie sur une dénonciation plus argumentée de "l'oppression jacobine" qui se traduit entre autres par des discriminations à l'égard des langues régionales : interdiction d'usage, refus de financer l'enseignement. Les mesures d'exclusions réglementaires envisagées par les mouvements indépendantistes pour renverser l'érosion de la langue ou pour promouvoir l'identité nationale, une fois le pouvoir conquis, indiquent de surprenantes convergences avec l'attitude du Front national : corsication des emplois ou préférence nationale? Plus généralement on peut se demander comment ces particularismes d'exclusion fonctionneraient s'ils avaient le champ libre, comment ils définiraient l'appartenance au nouveau peuple indépendant: y trouverait-on simplement des mécanismes électoraux de discrimination ou verrait-on une logique de purification ethnique "douce" se mettre en place ${ }^{20}$ Quoiqu'il en soit, l'adversaire naturel est la nation dominatrice, non une Europe dont la dimension culturelle n'existe pas.

11 La dimension européenne n'apparaît pas non plus avec beaucoup de relief chez nos acteurs sur le plan des relations entre organisations. Les mouvements séparatistes ont certes noué des liens entre eux, mais ces liens sont décalés par rapport à la construction européenne. Ils renvoient à un phantasme d'Europe méditerranéenne ${ }^{21}$ ou à une Europe des régions située aux antipodes de l'UEO ou de l'OTAN, dont la carte orne les bureaux des mouvements nationalistes depuis de longues années. L'enracinement dans une tradition de gauche où la dimension symbolique l'emporte largement sur les préoccupations d'action favorise la distance avec les choix diplomatiques européens présentés comme lourds d'implications : "Maastricht sonne le glas de la neutralité" dit le Sinn Fein et l'ANC remarque circulairement "que ce grave problème se pose d'ores et déjà en Irlande". Ces choix se concrétisent dans des structures hors souveraineté comme la Corse $\mathrm{e}^{22}$ dans des manifestations à caractère politico-culturel. 
12 La logique à l'oeuvre dans cette première configuration d'acteurs peut être qualifiée de militaire au moins au sens métaphorique du terme. Elle est faite d'affrontements et de conquêtes. Affrontements : attentats, violence polémique des discours, mobilisations des foules. Conquêtes: de positions institutionnelles certes, mais conçues comme une lutte pas à pas visant à créer par accumulation les conditions qui permettront le basculement vers l'autonomie ou l'indépendance. Dans cette logique, l'Europe n'existe que comme réalité inférieure, sa signification - super-État, simple élargissement de l'État-nation, ou plus modestement horizon géographique de l'ultra-libéralisme de l'Europe - est subordonnée à celle de l'État-nation Mais dans les trois cas, l'Europe "du fric et des flics" ${ }^{13}$ est le contraire des valeurs et du modèle de société souhaité par nos acteurs. Produite par une logique d'oppresseurs elle est oppressive : "Les Basques, les Corses savent depuis longtemps que la CEE n'a apporté aucune réponse à leur revendication légitime" ${ }^{24}$.

13 Cette absence d'Europe à l'horizon des organisations clandestines explique que celle-ci ne soit pas menacée. Dans le bouleversement international de la fin des années 90, le seul modèle perçu par les acteurs nationalistes n'a pas été la construction européenne, mais la désintégration de l'Europe de l'Est. Certes, des nationalités y ont obtenu leur indépendance, mais dans des circonstances historiques qui ne sont pas transposables à l'Ouest où l'effondrement du centre - en France, au Royaume-Uni, en Espagne non seulement ne s'est pas produit mais est improbable; en outre, à l'Est, la lutte armée clandestine n'a joué aucun rôle! La tentation indépendantiste chez les protestants que nous dépeint E. Picard en Irlande du Nord est de ce point de vue décalée. Si elle prenait corps elle serait probablement vouée à l'échec.

14 L'Europe n'a finalement servi aux organisations clandestines ou à leurs représentants légaux que de thème de propagande et de scène médiatique. Cet aveuglement s'explique probablement par des raisons de générations, de formations, de savoir-faire pour tout dire. Il y a probablement là un goulet d'étranglement pour des mouvements dont la structuration organisationnelle ou idéologique est figée sur les mythes du passé.

Concurrences triangulaires

15 Pourtant, à côté de la rhétorique combattante que nous venons d'analyser, le réalisme perce bien souvent car beaucoup comprennent que l'indépendance en Europe de l'Ouest n'est pas une réalité qui s'arrache mais un objet qui se construit, et qu'à des enjeux d'ordre institutionnel doit correspondre une démarche de même nature. Une deuxième configuration générale se dessine donc chez nos acteurs nationalistes. Elle regroupe des partis politiques - SPLD, PNV, Ligues lombardes, MPA et autonomistes corses -, pour lesquels la Communauté n'est pas seulement le substitut absent de la nation ou son lointain et pâle reflet, mais bien un enjeu véritable de la lutte politique.

16 L'Europe étant avant tout une Europe procédurale et juridique, c'est sur le plan de l'utilisation du droit et des institutions que ces acteurs se positionnent. La Communauté européenne présente de grandes possibilités pour des acteurs avisés, dans les domaines de la protection individuelle, économique, culturelle et enfin sur le terrain politique. Une logique d'avocat a fait prendre conscience des possibilités de recours contre les décisions de l'État qui existaient à la Cour européenne de justice ${ }^{25}$ et les unionistes trouvent tant de charmes à la Convention européenne des droits de l'homme qu'ils vont jusqu'à dire qu'elle devrait être incorporée à la loi fondamentale de l'Irlande du Nord ${ }^{26}$ ! Sur le plan économique et social, le comité des régions et la 
politique de cohésion sociale de la Communauté Européenne permettent d'espérer des subventions dont les partis régionalistes cherchent à maîtriser le mieux possible les mécanismes d'attribution, afin d'éviter que l'État-nation n'en fasse un moyen de pression. L'UPC souligne le besoin de "casser le filtre de l'État français" en établissant une liaison directe entre la Corse et Bruxelles, c'est aussi une des récriminations principales du gouvernement autonome basque contre Madrid $^{27}$. Sur le plan culturel, la Charte européenne des langues régionales et minoritaires mise en oeuvres par le Conseil de l'Europe est également un enjeu important des luttes institutionnelles parce qu'elle pourrait contraindre les États à reconnaître par ce biais "la réalité" d'un peuple, le peuple corse par exemple.

17 Sur un plan plus politique, la participation d'élus nationalistes au Parlement européen permet aussi de jouer un rôle. Le SDLP a ainsi été à l'origine du rapport Haagerup sur la situation en Irlande dont Ies recommandations furent adoptées en 1984 par l'Assemblée européenne. Ce dynamisme contraste avec l'attitude en retrait des acteurs que nous avons rattachés à la première configuration : députés du Sinn Fein, unionistes ou même Corses. Il a fallu attendre juillet 1992 pour que l'Assemblée de Corse propose la mise en place d'un correspondant permanent pour la préparation et le suivi des mesures et des programmes concernant la Corse à Bruxe1les ${ }^{28}$.

$18 \mathrm{Si}$, au départ, les interventions sur la scène européenne ont pu s'interpréter en terme de réactions instrumentales ou sectorielles dont les médiocres prestations de membres du groupe Arc en $\mathrm{Ciel}^{29}$, plus soucieux de stratégie médiatique que d'actions, donnent une idée, elles ont vite changé de nature. L'Europe, pôle autonome, dont les fins et les moyens ne concordent pas toujours avec ceux des États-membres, permet d'accumuler des ressources politiques à qui s'en donne les moyens. Elle a provoqué des regroupements, instauré une dynamique. Ennemis à Belfast, les trois députés européens d'Irlande du Nord coopèrent à Strasbourg, remarque E. Picard. Lan Paisley met entre parenthèses son opposition à la CEE pour siéger au comité d'agriculture du Parlement européen, même s'il ne croit pas comme les nationalistes constitutionnels ou les unionistes libéraux que les deux Irlandes seront rapprochées par la Communauté. Un certain nombre de partis tentent de se positionner sur le plan européen pour obtenir ensuite par effet de retour un avantage dans la compétition locale. Strasbourg a joué un rôle important dans la carrière de J. Hume, chef du SDLP, et dans le renforcement des positions électorales de ce parti en Irlande du Nord. Cette attitude mécontente d'ailleurs à la fois les unionistes nord irlandais, qui dénoncent le "romanish plot", et les nationalistes du Sinn Fein dont le candidat Danny Morrison a perdu pied aux élections devant un J. Hume auréolé de ses succès européens. Ce changement total $\mathrm{du}$ répertoire d'action, puisque la pratique des institutions européennes demandait un savoir-faire de juriste plus que de militant, de responsable politique plus que de chef d'organisation clandestine, se traduit par la place centrale qu'occupe désormais l'Europe dans la recherche d'une solution au problème de régionalisme et d'indépendantisme. A. Guelke reclasse les acteurs politiques en séparant ceux pour qui la construction communautaire est le seul cadre efficace, des mentalités "cloisonnées et restreintes" des autres. II montre bien qu'il y a corrélation entre le désir de désectariser les problèmes, voire de partager le pouvoir avec le clan opposé et l'adhésion à l'Europe. Dans cette perspective de concordance entre l'idéal d'une Europe unifiée et le cadre plus large de l'élimination des frontières et des divisions de l'Europe, la construction européenne permet d'éviter les pièges de l'ultranationalisme dont l'Est montre le désolant spectacle et de vérifier la possibilité des 
évolutions constitutionnelles sans tabou de frontière. Elle repose sur la croyance d'harmoniques entre Europe et régions: "Les tendances des processus démocratiques exigent une politisation démocratique au niveau macro et en même temps la facilitent, l'exigent au niveau régional et local ${ }^{30}$ écrit J. Hume. Le SDLP, perdu dans son rêve, allait jusqu'à imaginer une duplication des institutions de la $\mathrm{CE}$ pour résoudre le problème constitutionnel irlandais.

Derrière cet épisode, on touche au phénomène plus large de la constitution progressive sur le plan régional de groupes de professionnels de la politique disposant de pouvoirs institutionnels et de ressources et dotés d'une vision "stratégique" de l'avenir de leur région $^{31}$. Il peut s'agir d'une vision essentiellement économique cherchant à développer des potentialités industrielles ou commerciales, mais elle peut se doubler d'une vision culturelle comme c'est le cas au Pays basque par exemple, ou en Corse. Le cas de la Catalogne ou des Ligues lombardes se situent à mi-chemin entre les deux pôles. Ces processus sont en quelque sorte légitimés par le comité des régions que le traité de Maastricht a réaffirmé solennellement. Ils renvoient dans des contextes assez différents à une conscience d'Europe très variable : bien affirmée en Irlande du Nord qui en est le cas emblématique, plus faible au Pays basque où le PNV surtout engagé dans la politique d'autonomie locale, donc dans une compétition avec les autorités espagnoles, a une activité européenne en retrait, marginale en Corse où C. Lefebvre souligne à juste titre la place très limitée qu'occupent dans la classe politique locale les préoccupations européennes.

Ambiguïtés européennes

20 Il reste à mesurer la dimension corrosive de ces choix sur l'ensemble européen. Il n'y a, tout d'abord, pas de front unique entre les acteurs que nous avons examinés. Nos nationalistes pro-européens ont su tirer parti de l'échec de la lutte armée et se sont adaptés à la nouvelle donne politique et tout spécialement à la décentralisation. L'Europe représente pour eux le lieu d'un arbitrage extérieur permettant d'obtenir des garanties contre l'État oppresseur. La différence entre ce jeu triangulaire complexe de concurrence politique et l'affrontement binaire que nous avions d'abord isolé est si considérable qu'elle est source d'incompréhension ${ }^{32}$.

Ce sentiment est renforcé par la différence d'attitude à l'égard de la violence. Au Pays basque, la ligne de clivage entre ceux qui refusent et ceux qui acceptent d'user de la violence pour atteindre leurs objectifs est assez nette depuis la signature du Pacte Ajuria $\mathrm{Enea}^{33}$. Si elle est plus floue en Irlande et en Corse où avec des modalités très différentes la violence ne peut être réduite à l'activité d'organisations clandestines, c'est quand même par rapport à cette coupure"stratégique" que les nouveaux venus se situent. Les Ligues lombardes n'ont pris des contacts avec les autres mouvements régionalistes que sur cette base: elles ont noué des relations avec les autonomistes catalans mais ont récusé ETA à cause de sa violence ${ }^{34}$.

Entre nos deux types d'acteurs il n'y a pas continuité/articulation mais concurrence/ conflit. Concurrence électorale pour récupérer les 10 ou $20 \%$ de voix qui vont aux mouvements extrémistes, conflit symbolique pour substituer à la thématique de la lutte armée celle plus austère de la gestion politique. Comme cette dernière est privilégiée par le jeu institutionnel national ou européen l'évolution de la mouvance nationaliste peut se lire comme un passage de relais plus ou moins volontaire entre deux stratégies et deux personnels. Passage de relais entre Herri Batasuna et le PNV au Pays basque, entre le Sinn Fein et le SDLP en Irlande. En Corse, la lutte est plus confuse mais 
l'éclatement du FLNC et les distances prises par le MPA avec les membres de la coalition Corsica Nazione vont dans le même sens. Quelles que soient les nuances qu'il faille apporter à ce tableau, la construction européenne a bridé la violence, et offrant un lieu d'arbitrage extérieur a favorisé la négociation au détriment de l'action directe.

Si la coupure sociologique et méthodologique au sein du monde nationaliste est avérée, son sens politique est incertain. A court terme, la dimension dynamique et procédurale de leur action permet de concilier l'inconciliable, de maintenir l'objectif d'indépendance tout en le conjuguant dans l'espace européen, d'engranger les effets de la décentralisation des pouvoirs sans renoncer à la revendication nationale. Mais il reste un trou noir dans cette attitude car la rhétorique autour de l'Europe des régions ne renvoie à aucune réflexion sur les conditions d'établissement d'un État postmoderne dans l'ensemble européen, et ignore tout des enjeux de Maastricht. Est-il possible de réclamer la création d'un État national, c'est-à-dire la concentration de pouvoirs et l'instauration d'une frontière alors que la grande leçon de l'Europe est justement que le cadre national ne peut rester le lieu exclusif de la sociabilité politique. La prise en compte de la construction européenne, à terme, heurte la logique des nationalismes. Il nous semble illusoire, dit le FLNC-canal historique" de souhaiter une nouvelle construction politique remettant en cause les États-nations et de souhaiter un nouvel État fut-il corse ; il faut une souveraineté dans l'interdépendance européenne".

Nous parlions plus haut de la mise en place d'un nouveau personnel politique au niveau régional, les partis nationalistes s'inscrivent-ils dans cette grande rénovation des rapports entre l'État et la région, l'Europe étant simplement l'arène la plus propice pour négocier. $\mathrm{Ou}$ au contraire, l'Europe des régions relève-t-elle d'une croyance téléologique naïve dans laquelle l'histoire gommerait les différences d'échelle en faisant converger les évolutions jusqu'à ce que petites régions et Etats-nations coïncident ${ }^{35}$; à moins qu'elle ne renvoie à une volonté instrumentale où l'Europe ne serait plus que le lieu vide où s'exercerait un arbitrage au détriment des États nationaux? Cette dernière hypothèse n'entraîne aucun sentiment de loyalisme ou de responsabilités collectives, mais au contraire favorise la réaffirmation identitaire au niveau régional et sa fermeture sur des mobilisations égoïstes. Entre le marchandage intéressé et la négociation politique, entre l'inscription identitaire crispée et la solidarité politique, la frange la plus ouverte du mouvement nationaliste n'a pas encore clairement choisi, il conviendrait qu'elle le fasse vite si on veut éviter que sous couvert d'une Europe des régions, mythique ou instrumentale, ne s'opposent l'Europe des "Singapour internes"36 - Lombardie industrielle, Catalogne marchande, Ecosse pétrolière - cherchant à optimiser leurs atouts et refusant la solidarité ; et celle des régions pauvres aspirant à se protéger derrière des règlements, à bénéficier des redistributions communautaires, pratiquant une stratégie de marginalité pour atteindre une hypothétique "autarcie nationalitaire" entretenue de l'extérieur ${ }^{37}$. 


\section{NOTES}

1. Les encouragements de Ligues Lombardes à l'indépendance slovaque illustrent cette coïncidence entre le "prénationalisme" de l'Est, et le"post-nationalisme de l'Ouest. 2. Les remarques angoissées de nos hommes politiques sur la catastrophe à l'Est relèveraient moins dans cette hypothèse de la philanthropie que de la prophylaxie. 3. Ce numéro n'a pas pour vocation de couvrir l'ensemble des problèmes régionaux en Europe, par exemple nous ne parlons pas du problème catalan ou de la situation interne en Belgique. Il s'agit de fournir quelques exemples significatifs.

4. Cela paraît assez normal pour les mouvements autonomistes enracinés dans le local, mais par contre c'est plus surprenant pour les partis d'extrême droite dont l'idéologie aurait dû justifier un intérêt plus profond.

5. Voir l'article d'A. Chebel d'Appollonia, "Les partis d'extrême-droite et I'Europe".

6. Ibidem.

7. Voir l'article de C. Lefebvre, "Nationalismes corses et perspectives européennes".

8. Voir A. Guelke, "Les catholiques en Irlande du Nord face à l'Europe".

9. Voir E. Picard, "Les protestants en Irlande du Nord face à l'Europe".

10. Voir D. Hermant, "La question basque au miroir de la violence".

11. Il n'y a pas unanimité au sein du SPLD comme le montre l'attitude de deux membres importants du parti Garry Fitt et Paddy Devlin en 1975 lors du vote sur l'adhésion du Royaume-Uni à la CEE.

12. A. d'Appollonia cite ce slogan du parti Republikaner dans la campagne pour le Parlement européen : l'Allemagne d'abord, l'Europe ensuite !".

13. L'idéologie du Front national défend le "particularisme régional" dans la mesure où celui-ci constitue un approfondissement des racines identitaires, et s'oppose au "régionalisme radical" visant à démembrer la nation.

14. C'est sur cette question que le FLNC-canal historique et le FLNC-canal habituel se séparent! Au Pays basque, la question a été tranchée par la prééminence de l'ETA sur le mouvement nationaliste, mais cette prééminence semble-t-il s'affaiblit.

15. La géographie des attentats des organisations clandestines à vocation nationalitaire et indépendantiste connaît deux cercles : le premier régional (Corse, Pays basque, Irlande du Nord), le second national ("continent", Espagne, Grande-Bretagne). Les seuls attentats "internationaux sont ceux que l'IRA a commis contre les troupes britanniques stationnées en Allemagne.

16. Voir l'article de C. Lefebvre.

17. Voir l'article de D. Hermant.

18. Voir l'article de C. Lefebvre.

19. Voir entretien. L'identité est définie par rapport à l'émigration et non par rapport à la langue.

20. Une des raisons qui a poussé l'ANC à voter non au référendum est le droit de vote donné aux ressortissants communautaires "alors que le corps électoral corse n'est pas reconnu".

21. Voir l'article de Christian Lefebvre.

22. Confédération des nations sans État de l'Europe occidentale, la CONSEO organise une fois par an les journées de Corse regroupant des mouvements nationalistes révolutionnaires - tendance LLN en Corse, Sinn Fein, Herri Batasuna. 
23. La formule est du mouvement basque libertaire Oldentzen.

24. EMA. Tout en refusant la logique de Maastricht, ce mouvement nationaliste de gauche tient quand même à rappeler ses convictions européennes.

25. Elle peut condamner les Etats comme cela est arrivé à la France le 27 août 1992. Voir

C. Lefebvre.

26. Voir E. Picard.

27. Voir entretien avec le PNV.

28. Voir C. Lefebvre.

29. Le groupe Arc en Ciel est intéressant à plus d'un titre, il ne peut faire l'objet d'un commentaire global parce qu'il est constitué de formations très différentes Mouvement populaire danois contre l'Europe, Verts allemands, Ligues lombardes, Parti national écossais, UPC.

30. Voir A. Guelke.

31. Voir le numéro 60 de la revue Pouvoirs, 1992

32. La méfiance envers l'Europe du Sinn Fein et les unionistes protestants met en évidence la symétrie des inquiétudes chez les acteurs les plus violemment engagés dans la lutte partisane : le Sinn Fein se méfie d'une Europe qui risque de faire échouer sa tentative pour réunifier l'Irlande, alors qu'au contraire les unionistes pensent que l'Europe menace de limiter la marge de manoeuvre anglaise et de favoriser la réunification.

33. Voir D. Hermant.

34. Voir entretien avec les Ligues lombardes.

35. Le PNV envisage une égalisation progressive des deux niveaux, les autonomies régionales s'élargissant alors que les pouvoirs des Etats se réduiraient. Voir entretien avec le PNV.

36. Expression de Paul Thibaud in J.M. Ferry et P. Thibaud, Discussion sur !'Europe, Paris, Calmann-Levy, p. 69.

37. Comme le montre la grande manifestation pour le statut fiscal dérogatoire qui a réuni toutes les tendances politiques en Corse le 28 novembre dernier.

\section{RÉSUMÉS}

L'Europe n'est-elle pas en train de faire le lit des contestations nationales ou micro-nationales, d'ouvrir la boîte de Pandore des nationalismes ? Cette affirmation peut paraître paradoxale, surtout au moment où face à la désintégration de l'Europe communiste il semble raisonnable de se féliciter des succès de l'intégration européenne. Paix à l'Ouest, guerre à l'Est donc, mais derrière cette rhétorique un peu trop affirmée ne faut-il pas lire une secrète inquiétude, comme si entre ces deux évolutions, existaient des passerelles voire une logique commune ? Que nous montre l'Est : un échec économique spectaculaire, doublé d'un changement brutal de système politique qui entraîne d'abord la décomposition du bloc impérial soviétique ; puis dans une deuxième étape provoque l'éclatement des États successeurs ravalés au rang de micro-empires ! Dans cette réduction progressive des échelles, dans ce jeu sans cesse renouvelé des logiques 
identitaires dont on se demande l'issue, ne peut-on trouver un principe général qui pourrait à l'occasion fonctionner à l'Ouest?

There is a growing tendency to analyse nationalism as a new plague creating chaos in Eastern Europe and the former USSR. Some speak of nationalism as a " natural " force. But we must remember that nationalism is always constructed by " identity entrepreneurs " and thus deeply depends on the political context. If many studies have been published concerning nationalism in Eastern Europe, none have discussed the influence of this " renewal " for the nationalistseparatist movements in the European Community. Is there a " contagion " of nationalism and fragmentative dynamics capable of challenging the national states at the time they are also being challenged by the construction of the EC, or will the EC be the necessary " intermediary " between the national states and certain aspirations for more autonomy?

\section{INDEX}

Index géographique : Europe

Mots-clés : Nationalismes, construction européenne

\section{AUTEUR}

\section{DANIEL HERMANT}

Professeur agrégé d'histoire, directeur de publication de Cultures \& Conflits. 